\title{
Dynamic Policy Management on Business Performance Management Architecture
}

\author{
Teruo Koyanagi ${ }^{1}$, Mari Abe ${ }^{1}$, Gaku Yamamoto ${ }^{1}$, and Jun Jang Jeng ${ }^{2}$ \\ ${ }^{1}$ IBM Tokyo Research Laboratory, \\ Yamato-shi, Kanagawa-ken, Japan \\ \{teruok, maria, yamamoto\}@jp.ibm.com \\ ${ }^{2}$ IBM T.J. Watson Research Center, \\ Yorktown Heights, New York, United States \\ jjjeng@us.ibm.com
}

\begin{abstract}
Business performance management (BPM) is a new approach for an enterprise to improve their capabilities for sensing and responding to business situations. In a diverse and fast-changing business environment, an enterprise needs to adapt itself to any unexpected changes. For BPM, such changes imply changes of the models and services that support BPM. This paper discusses an implementation of BPM with the focus on dynamically adapting its services. We will present the motivation, concept and architecture of the dynamic change mechanisms. First we define a set of configurations as a policy, and also define its consistency through an application context. Then we propose an architectural overview including a policy management service as an implementation of consistency management.
\end{abstract}

\section{Introduction}

Business performance management is a new approach for an enterprise to improve their capabilities of sensing and responding to business situations [1]. Its functionality covers data capturing, metric realization, situation detection, decision making, action rendering and business analytics. Business performance management evolves from business process management that is aimed to capture enterprise behavior in a canonical form and to automate encoded operations as much as possible. Business processes themselves are realized utilizing service based process modeling languages, such as BPEL4WS [2]. While business process management is focused on operational aspects, business performance management puts more emphases on improving the quality of an organization. In this paper, we use the BPM to refer business performance management. Similar to business process management systems, BPM systems can be implemented in many ways. We have found Service Oriented Architecture (SOA) [3] is particularly suitable for building management services and BPM systems.

In a diverse and fast-changing business environment, an enterprise needs to adapt itself to any unexpected changes and make corresponding adjustment in the areas such as processes, IT systems etc. For the area of BPM, such changes imply the dynamic changes of the models and services that support BPM. Consequently, BPM system is expected to cope with the changes and change itself correspondingly. The 
characteristics of SOA such as modularity and adaptivity make itself a good architectural principle upon which the BPM system can be built.

The concepts of dynamic change management are discussed in previous works [4, $5,6,7]$. They include the idea of maintaining consistency during changes. Their concepts of consistency are considered to be guaranteed if the modified component is not included in the execution of any transaction context. Most of these works define their consistency criteria on the basis of explicit inter-component interactions, and then propose management methods of dynamic software evolution to maintain the consistencies. However, the fact that it is difficult to maintain consistency among a number of configurations within an application context hinders most efforts. Such difficulties would come from the loosely-coupled services which do not have the explicit interaction that has been defined among them although those services may have semantic relationships through the application context.

In this paper, we would like to describe the mechanism of using policies to manage BPM systems. We use the BPM as our focus area in this paper although the technology itself is generic. It can be applied to another service-oriented computing platform [8] that is aimed to maintain its consistency while changing configurations of services without stopping the system.

Firstly, we briefly introduce a dynamic reconfiguration for a BPM architecture in Section 2. Then we explain concepts of dynamic policy management in the BPM architecture in Section 3. The architectural overview of managing the consistencies of BPM services is shown in Section 4 and we conclude this paper in Section 5.

\section{Dynamic Reconfiguration for a BPM Architecture}

A BPM system is built as an SOA with supporting functions such as monitoring for business metrics and for detecting situations, and abilities to perform actions. All of the BPM services can be configured dynamically based on new change requirements from the business demands. Also, a lot of application contexts are generated to monitor various points of views of business situations. In this section, an example and the concept of dynamic reconfiguration of BPM applications based on policies are described.

Let us consider an order process that needs inventory management as an example, such as a PC order process from the Web. Stock for each model of PC is prepared based on how many PCs are ordered in certain interval. In this application, as shown in Fig. 1, three components of the services are used. Monitor is the component to observe the counts of the ordered items. Forecast Provider is also a key performance indicator (KPI) calculator to predict consumption for a given strategy. Inventory Manager provides an action to keep sufficient stocks to respond to the predicted consumption.

Initially, all orders from customers are considered with equal weight of service level for preparing stock. Therefore, the Monitor records all customers' orders into one set of counts, and the Forecast Provider gives one set of predictions. However, in fact, customers are categorized into two different types: the gold customers who request high-performance machines and the regular customers who request low-cost machines. 


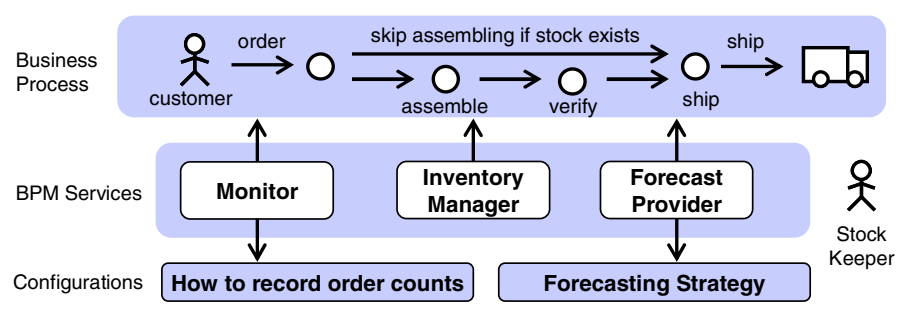

Fig. 1. An example of a BPM system: a PC order process

To respond to the gold customers' demands to be prioritized, it is necessary to change the monitoring method and the forecasting strategy to be biased for gold customers. However, the change must be applied without stopping the system, because the service should be provided 24 hours a day, 365 days a year. Additionally, even though both services are provided independently, their configurations are dependent on the application context, in which the order counts is observed and consumption is predicted based on the counts.

This is a typical case in which an external management function, called dynamic policy management, is required to maintain consistency among configurations.

\section{Dynamic Policy Management}

In the previous section, BPM applications are customized to respond to business policies. Such a customization consists of a number of configurations which are deployed into actual components of services. It implies that the BPM system is desired to support functions to handle such a configuration set dynamically.

One of the desired functions to dynamically adapt BPM applications to business policies is a runtime consistency management. For instance, it is the case of inconsistent that an event is issued after changing its producer to introduce a new format before its consumers have not changed yet for the new one. Basically, when a configuration is changed, it is necessary to avoid activating of the changed component during transition. In addition, we need to consider cases that configurations of multiple components are changed. In such case, the dependent configurations must be applied atomically for the application context which employs them.

In this section, we present a dynamic policy management function that maintains the consistency of configurations by categorizing them into three levels of a runtime application context.

A policy is a general rule about how to operate a BPM application in accordance with a business demand [1]. It is broken down into a set of configurations of the BPM services used in the application. It is important that the configuration set is defined externally as a policy. BPM services are provided by corresponding components in the BPM system.

A configuration is a component which is executed to changes a set of parameters or externalized logics of another component (called customized component). Configurations are identified by names which are associated with customization 
points of components, and they are also labeled with versions to identify them in the change history. A consistency version set includes versions of configurations to specify which changes need to apply consistently.

The application context is an execution path including the sequence of service employments which provide a function to business users based on a business policy. To be consistent with policy, the customized components which provide the services in an application context have to be configured with the same configuration set. Therefore the consistency among a set of configurations is defined by considering the ranges of the application context.

While applying configurations, the states of the customized components must be guaranteed not to be executing in any application contexts. Because changing a configuration in the middle of the context provides a chance to change the dependent artifacts of the rest of execution. It causes a malfunction behavior.

In addition, the relationships among configurations must be also guaranteed in an application context. In the BPM architecture, these relationships come from business policies. Thereby, in this paper, we categorize the consistency into three cases by the level of expansion range of the application context, as shown in Fig. 2

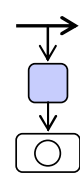

a) component-local

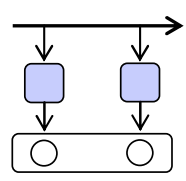

b) context-wide

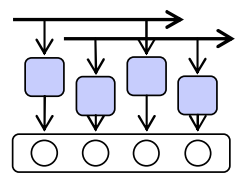

c) inter-context

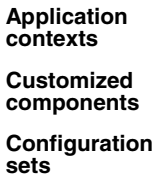

sets

Fig. 2. Types of consistency of configuration sets

For a), component-local-consistency is guaranteed if a configuration transaction is completed while the configuration is not currently executing in any application context. This level of consistency is mandatory for dynamic reconfiguration, and b) and c) are the options used to preserve application semantics.

For b), context-wide-consistency is guaranteed if component-local-consistency is maintained for each configuration used in the application context, and based on it, a set of configurations which implements a policy is used together in an application context. In this case, consistency is represented by a configuration set which is provided externally. This is necessary, for example, the set of configuration which includes the Monitor's configuration and Forecasting strategy must be used together during an application context which is an interval of stock preparation described in Section 2.

For c), inter-context-consistency is guaranteed if context-wide-consistency is maintained for each context, and in addition, the entire application context that is running concurrently is configured with the same set of configurations. In the example of the PC order process, it is assumed that there is an interval for each model of PCs. Because the configuration change invoked by a drastic policy change, such as adding a new axis of customer type, affects all the application contexts, the changes must be applied to them synchronously. 


\section{Architectural Overview}

As described in the previous section, a consistency version set represents a set of constrained configurations that must be applied to the components executed in the same application context. It is defined at design time, but at runtime it does not depend only on the static component structures, but also on the dynamic characteristics of the application context as described in the previous section. In this section, a framework to maintain consistency of the consistent version set within an application context is described. This can be considered as a hybrid method between dynamic relationship management in application contexts, and atomic configuration deployment that maintains the consistency of the configuration set.

The policy management service provides a runtime management function for configuration sets. This service consists of the following components: session factory, configuration registry, and version coordinator, as shown in Fig. 3.

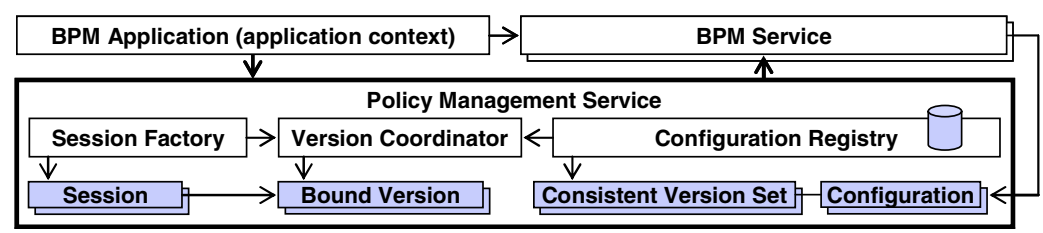

Fig. 3. Architectural overview of the dynamic policy management

The application which employs the customized components creates a session for each application context correspondingly. According to the sessions, the right configurations are provided to the customized components in the context.

The configuration registry maintains registered information of the consistent version sets which represent a kind of dependencies defined at design time. As a function of the configuration registry, the new configurations and consistent version sets can be registered in the registry without stopping the system. Retaining the current versions is another responsibility of the configuration registry. The configuration registry provides an administrative interface to deploy configuration sets and to specify current versions.

When a configuration is used in the session firstly, the current version of the configuration and its constrained versions are associated with the session. When the other configuration is used after that, at first it is searched from the associated versions. If it does not exist, it is requested to the configuration registry. The version coordinator maintains association between the session and versions of configurations.

\section{Conclusion}

In a diverse and fast-changing business environment, an enterprise needs to adapt itself to any unexpected changes. In the areas of BPM, in order to adapt alreadydeployed BPM applications to such business environments, it is imperative to provide dynamic reconfiguration capabilities. The BPM system is build as an SOA with 
supporting functions for tasks such as monitoring business metrics, detecting situations, and performing actions. Therefore an application in the BPM system is customized to adapt to a business policy by configuring each service component.

In this paper, a policy is defined as a configuration set, which is deployed into actual components. Consistency management of the configuration set is a key to realize the dynamic reconfiguration, thereby we categorized the consistency into three cases based on the level of expansion range of the application context; componentlocal, context-wide and inter-context. Then we proposed an architectural overview including a policy management service as an implementation of consistency management.

To allow business users to change business policies, it is necessary for BPM system not only to provide the runtime mechanisms but also to support designing and deploying business policies. In the future work, we will investigate what kind of supporting method can be provided.

\section{References}

1. Jeng, J.J., Chang, H., Bhaskaran, K.: On Architecting Business Performance Management Grid for Adaptive Enterprises. In Proceedings of the 2005 Symposium on Applications and the Internet (SAINT' 05). (2005) 110-116

2. Thatte, S. et al.: Process Execution Language for Web Services Version 1.1. ftp://www6. software.ibm.com/software/developer/library/ws-bpel.pdf (2003)

3. Papazoglou, M.P.: Service-Oriented Computing: Concepts, Characteristics and Directions. In Proceedings of the Fourth International Conference on Web Information Systems Engineering, IEEE Computer Society (2003)

4. Kramer, J., Magee, J.: The Evolving Philosophers Problem: Dynamic Change Management. IEEE Transactions on Software Engineering 16 (1990) 1293-1306

5. Warren, I., Sommerville, I.: A Model for Dynamic reconfiguration which Preserves Application Integrity. In Proceedings of the 3rd International Conference on Configurable Distributed Systems (ICCDS'96), IEEE Computer Society (1996) 81-88

6. Chen, X., Simons, M.: A Component Framework for Dynamic Reconfiguration of Distributed Systems. In Proceedings of the IFIP/ACM Working Conference on Component Deployment. Volume 2370. (2002) 82-96

7. Truyen, E. et al.: Dynamic and Selective Combination of Extensions in Component-Based Applications. In Proceedings of the 23rd International Conference on Software Engineering (ICSE'01). (2001)

8. Lazovik, A., Aiello, M., Papazoglou, M.: Associating Assertions with Business Processes and Monitoring their Execution. In Proceedings of International Conference on Service Oriented Computing (ICSOC'04). (2004) 94-104 